\title{
La división del trabajo como una relación socio-espacial, o cómo reconciliar la ciencia económica y la geografía ${ }^{1}$ The division of labor as socio-spatial relations, or how to reconcile economics and geography
}

Journal of Economic Literature (JEL): $R 1, J 23,011, R 23, R 58$

\section{Palabras clave:}

Ciencia económica

Geografía económica

División del trabajo

Desarollo desigual

Keywords:

Economics

Economic Geography

Division of Labor

Uneven Development

\section{Resumen}

En la ciencia económica no se presta la atención suficiente al espacio y a la geografía. En este documento se plantea que la idea de la división del trabajo es adecuada para superar esta omisión y para re-insertar el pensamiento geográfico en el análisis económico. Cada división del trabajo se desarrolla en y mediante una yuxtaposición espacial de las actividades económicas, por lo que se define a partir de un conjunto de relaciones y procesos socio-espaciales en varias escalas geográficas, entre otras, la escala del hogar, de la ciudad y de la economía mundial. Sin embargo, un análisis comprensivo de la división del trabajo, entendiendo ésta no como un principio abstracto, sino como una práctica económica real, no sólo revela la geografía de la economía, sino también que se trata de una herramienta poderosa para analizar las relaciones de producción. Queda claro que toda división del trabajo consiste en un conjunto de relaciones socio-espaciales, y que mediante estas relaciones socioespaciales es que las actividades económicas, los grupos sociales que las realizan y los lugares en los que las hacen son jerarquizados. Por ende, en la división del trabajo transfronteriza el desarrollo desigual es resultado de una integración diferenciada de personas o sociedades y de sus lugares o regiones.

1 Este texto forma parte de los esfuerzos institucionales realizados por la Facultad de Economía para reforzar la relevancia de la perspectiva geográfica en la ciencia económica. Durante mi estancia en la Facultad, hecha posible por el Servicio Alemán de Intercambio Académico (DAAD por sus siglas en alemán), se desarrollaron varias actividades al respecto. Una de ellas es un libro de texto sobre geografía económica que será publicado por la misma Facultad, otra es este documento. Agradezco la bienvenida que me han brindado los miembros de la Facultad, el apoyo económico del DAAD y de la Dirección General de Cooperación e Internacionalización de la UNAM, así como la ayuda de Goneril Paloma Gutiérrez Sifuentes y de Jorge Luis Ruiz en la elaboración de este documento. 


\section{Abstract}

In economics, not enough attention is being paid to space and geography. In this document I claim that the idea of the division of labor is well suited to overcome this shortcoming and to re-insert geographical thinking in economic analysis. Each division of labor takes place in and is developed through spatial juxtapositions of economic activities, and that is why it consists of socio-spatial relations and processes at various geographical scales, including the home, the city and the global economy. However, a comprehensive analysis of the division of labor, understood not as an abstract principle, but as a real economic practice, not only reveals the geography of the economy. Rather, it is also a powerful tool for analyzing relationships of production. Any division of labor consists of a set of socio-spatial relationships, and it is through these socio-spatial relationships that economic activities, social groups that perform them, and the places in which they so it, are hierarchized. Therefore, in the cross-border division of labor uneven development is the result of a differentiated integration of persons or societies and their locations or regions.

\section{Introducción}

En un libro de reciente aparición sobre el rol que desempeña el espacio en el pensamiento económico,

Asuad Sanén $(2014,34)$ se resume que en la ciencia económica "la concepción de espacio y su vinculación con la actividad económica, prácticamente es desconocida y simplificada". Del mismo modo, Valdivia López y Delgadillo Macías $(2013,11)$ hacen constar que "tanto geógrafos como economistas, a pesar de estar discutiendo e investigando temas semejantes desde una perspectiva regional, mostraban en lo general poco interés en discutir entre ellos mismos sobre qué los podría identificar como investigadores sociales". Tales evaluaciones críticas de las no-relaciones entre la ciencia económica y la geografía no son nuevas. Es bien conocida la observación burlona de Isard $(1956,25-26)$ cuando dice que los economistas se instalaron cómodamente con sus teorías y modelos en un "wonderland of no spatial dimensions [...] a one-point world", en el que "all factors and producers, commodities and consumers are, in effect, congregated at one point".

Sin embargo, ¿no han cambiado las cosas con la "Nueva Geografía Económica" que según su representante más conocido, Paul Krugman (1998, 7), "serves the important purpose of placing geographical analysis squarely in the economic mainstream"? Los geógrafos, por lo menos, se muestran poco convencidos. Para Martin $(2013,235)$, “(e)sta Nueva Geografía Económica [...] no es tan nueva, ni es Geografía”. Que esta noción no es exagerada se ve también en la ausencia absoluta de perspectivas espaciales en los libros de texto de macroeconomía. En el libro de texto del propio Krugman (Krugman / Wells 2012), por ejemplo, no existe ni una entrada en el índice para Geografía, Espacio, Región, Aglomeración o Ciudad. De hecho, Krugman parece 
La división del trabajo, entendida como una práctica económica real

y no como un principio abstracto, implica al concepto de geografía ser consciente de la debilidad de su proyecto, cuando reconoce que "this name (New Economic Geography) can annoy hard-working traditional economic geographers, who feel both that many of the things the 'new' geographers say are old hat, and also that new economic geography ignores almost as much of the reality they study as old trade theory did"' (Krugman 2000, 50, énfasis añadido).

La omisión de la geografía en el pensamiento económico puede sorprender, ya que la ciencia económica está equipada con herramientas analíticas que tienen el potencial de llamar la atención sobre la espacialidad inherente de la economía. De manera particular me refiero al concepto de división del trabajo como principio fundamental de la organización económica. Desde los filósofos griegos hasta los análisis sobre la globalización se observa que las reflexiones sobre los beneficios económicos que empresas y sociedades pueden obtener a partir de la división del trabajo, tanto dentro de la firma como entre ellas, ocupan un lugar destacado en el pensamiento económico (Sun 2012). No obstante, los economistas no han explotado a plenitud el potencial de un análisis de la división del trabajo, ya que sus observaciones, en la mayoría de los casos, han sido limitadas, en el sentido de que se han enfocado en las dimensiones funcionales y sociales de la división del trabajo, mientras que la dimensión geográfica relacionada con estos procesos no ha recibido la atención apropiada.

Ésta precisamente es la idea central de este análisis: que la división del trabajo, entendida como una práctica económica real y no como un principio abstracto, implica al concepto de geografía. La fragmentación de un proceso de producción, la especialización de ciertos productores o trabajadores en ciertas labores, así como la re-integración última de las secciones para obtener un producto final no pueden tener lugar en la "punta de la aguja" proverbial, porque allí literalmente no caben. Las relaciones y procesos que forman y sostienen la división del trabajo siempre son desarrolladas en espacios concretos y por eso diversos, que se utilizan para darles forma y características específicas. Por ende se trata de relaciones y procesos socio-espaciales en varias escalas geográficas, del hogar, de la empresa, o de la ciudad o región, o incluso hasta en la escala de la economía mundial. Más aún, identificar el patrón espacial de las relaciones sociales de la división del trabajo rebasa una simple mirada descriptiva. Un examen de las geografías de las relaciones de producción es una herramienta poderosa para su análisis porque hace reconocible las propiedades de las divisiones del trabajo, a saber, su carácter jerárquico. El desarrollo desigual que caracteriza el desarrollo económico a varias escalas (de la segregación socio-económica en las ciudades, hasta la división Norte-Sur a nivel global) es, en síntesis, el resultado de una integración diferenciada de personas o sociedades, y sus lugares o regiones en la división del trabajo transfronteriza.

\section{División del trabajo en el hogar}

El filósofo griego Xenophon, cuyo texto Oikonomikos es considerado el primer escrito sobre economía, hace énfasis en que la administración del hogar requiere de la división del trabajo. Dice que esposo y esposa, aunque comparten la propiedad, tienen tareas distintas, tal como los esclavos tienen quehaceres propios, diferentes a los de los amos. Xenophon describe también con claridad la geografía 
relacionada con estas divisiones del trabajo. Según él, la administración del hogar no sólo implica que se asignen tareas diferentes a las personas, sino también que las personas ocupan espacios diferentes para realizar sus actividades. En cuanto a la división del trabajo por género anota:

Those who intend to obtain produce to bring into the shelter need someone to work at the outdoor jobs. For ploughing, sowing, planting, and herding is all work performed outdoors, and it is from these that our essential provisions are obtained. As soon as these are brought into the shelter, then someone else is needed to look after them and to perform the work that requires shelters. The nursing of newborn children requires shelters, and so does the preparation of bread from grain, and likewise, making clothing out of wool. Because both the indoor and the outdoor tasks require work and concern, I think the god, from the very beginning, designed the nature of woman for the indoor work and concerns and the nature of man for the outdoor work. (Citado en Sun 2005, 39; énfasis añadido)

De igual manera, para Aristóteles la economía implica una división del trabajo, y acentúa la dimensión espacial de esta división del trabajo. Pastores, agricultores, pescadores, cazadores e incluso bandidos tienen sus propios espacios -la actividad económica del pescador obviamente necesita del río, la del pastor el pasto, etcétera. Más aún, Aristóteles señala la importancia de conocer estas geografías. Dedicarse a la ganadería requiere, por ejemplo, conocimientos

(of) which (husbandry) are most profitable, and where, and how as, for example, what sort of horses or sheep or oxen or any other animals are most likely to give a return. A man ought to know which of these pay better than others, and which pay best in particular places, for some do better in one place and some in another. (Citado en Medema / Samuels 2003, 11; énfasis añadido)

Tanto Xenophon como Aristóteles señalan que las divisiones del trabajo más fundamentales, las observadas en la familia y en el pueblo, no sólo son funcionales (entre labores) y sociales (entre personas), sino que las actividades y las personas que las realizan también están vinculadas a lugares específicos. Sugieren, entonces, que la división del trabajo consiste en relaciones socio-espaciales.

Estudios arqueológicos, antropológicos e historiográficos apuntan en la misma dirección. Subrayan que las divisiones del trabajo por género siempre han tenido sus geografías particulares. Cuando la economista Matthaei $(1999,593)$ resume que "men's work centred in interfamilial activities, and women's in child-rearing and intrafamilial work", se refiere a las escalas de las actividades o al alcance geográfico en que tienen lugar: mientras que interfamiliar implica actividades fuera de la casa, intrafamiliar significa dentro del hogar. Del mismo modo las historiadoras Scott y Tilly (1975, 44-45) apuntan para las sociedades europeas pre-industriales que: "Men and women not only performed different tasks, but they occupied different space". En resumen, la división del trabajo en el hogar, que por mucho tiempo fue la unidad básica de la economía, involucraba tres elementos: primero, la división funcional del trabajo, 
o sea, la partición del trabajo necesario para la reproducción de la familia en varias tareas. Segundo, estas actividades eran efectuadas por actores diferentes -la división social del trabajo consiste en que personas o grupos diferentes se especializan en, o son asigandos a tareas particulares. Tercero, dividir el trabajo necesario para la reproducción familiar y asignar ciertas actividades a determinadas personas también implica crear espacios diferentes de trabajo. Mientras la casa y su entorno se convirtieron en el mundo laboral de la mujer, los hombres hicieron del mundo externo al hogar su territorio. El tercer elemento en la organización económica del hogar es, por ende, la división geográfica del trabajo. En la siguiente sección analizaré esta trinidad analítica a nivel de otra escala, la de las relaciones ciudad y campo.

\section{División del trabajo entre ciudad y campo Para Adam Smith la di-} visión del trabajo en la industria urbana es, como bien se sabe, la fuente principal de la Riqueza de las naciones (1776). Smith también reconoce que esta división del trabajo depende de la producción de un excedente en el campo. En consecuencia, la más fundamental división del trabajo que existe es la que se observa entre el campo y la ciudad: "The town, in which there neither is nor can be any reproduction of substances, may very properly be said to gain its whole wealth and subsistence from the country. [...] It is the surplus produce of the country only, or what is over and above the maintenance of the cultivators, that constitutes the subsistence of the town, which can therefore increase only with the increase of this surplus produce" (Smith, 1776, 364).

La ciudad para su desarrollo económico requiere, entonces, de las relaciones comerciales con el campo, al igual que el campo necesita, según Smith, a la ciudad. Con eso, Smith ofrece una conceptualización espacializada del desarrollo económico; es consciente de que las relaciones entre ciudad y campo son necesarias para el aprovechamiento de las diferencias entre las dos "entidades". Esta noción la reencontramos, aunque con peso analítico diferente, en muchos otros autores. Para Carlos Marx las relaciones entre ciudad y campo también son fundamentales para el desarrollo económico. Él apunta que: "La base de toda división del trabajo desarrollada, mediada por el intercambio de mercancías, es la separación entre la ciudad y el campo. Puede decirse que toda la historia económica de la sociedad se resume en el movimiento de esta antítesis" (Marx 2009a, 429). Por el otro, el historiador francés Fernand Braudel no se cansa de subrayar que la agricultura siempre desemboca en la ciudad. Al enfatizar lo obvio, este autor llama la atención sobre el hecho de que la expansión de las ciudades y el impulso que dieron -y dan- a la economía en general se basa en una división del trabajo geográfica, a saber la existente entre la ciudad y el campo: "In fact town and countryside never separate like oil and water. They are at the same time separate yet drawn together, divided yet combined. [...] In fact the countryside had to support the town if the town was not to live in a constant state of anxiety with regard to its subsistence" (Braudel 1985, 486-487; énfasis añadido).

La razón por la que ninguna ciudad puede existir sin la división del trabajo entre ella y el campo es porque de este último obtiene sus dos recursos fundamentales: alimentos y mano de obra. Las ciudades no sólo se alimenta(ba)n de comida proveniente 
del campo, sino también de la mano de obra que hacia ellas fluía -y fluye- de las zonas rurales. Baste aquí citar un ejemplo: en la Europa del siglo XII, cuando el floreciente comercio empezó a despertar a las ciudades hasta entonces desmejoradas, se necesitaba a los migrantes para avivar las economías urbanas, porque desde el punto de vista de su reproducción natural, las ciudades europeas pre-industriales "were large graveyards" (Cipolla, 1993, 104). Portes $(2000,153)$ precisa que: "Cities were the cradle in which Western capitalism originated almost a millennium ago, and migration to them fuelled their growth and their subsequent economic and political development".

Conocer los rasgos geográficos de las divisiones del trabajo no es un objetivo en sí mismo. Es una reivindicación central de la geografía económica que Identificar la el análisis del patrón espacial de un fenómeno o de un proceso social ubicación geográfica de cada tarea en una revele su propio carácter. Por ende, identificar la ubicación geográfica de división del trabajo cada tarea en una división del trabajo, así como reflexionar sobre el porqué ciertas tareas están localizadas en ciertos lugares corresponde a un análisis "(of) the spatial organisation of relations of production" (Massey, $1995,7)$. Este aspecto ni es trivial ni una tarea adicional voluntaria, sino clave para entender las reestructuraciones incesantes del capitalismo y su significado, porque estos cambios en las estructuras socio-espaciales responden a los cambios en las relaciones de clase a nivel nacional e internacional: el desarrollo desigual tiene su origen en una valoración sistemáticamente diferente de las actividades económicas, lo que resulta en la jerarquización de las actividades, de los actores que las realizan y de los espacios en los que los actores las llevan a cabo.

\section{División y jerarquización de trabajo (I): el hogar}

Investigaciones antropológicas e históricas indican que las divisiones del

trabajo funcionales, sociales y geográficas en el hogar no siempre estuvieron marcadas por desigualdades y jerarquías. Xenophon, por ejemplo, considera al esposo y a la esposa como socios en la administración de la casa. Aunque su texto refleja una relación patriarcal, en lo que se refiere a la economía de la casa, hombre y mujer son vistos como iguales. El análisis tanto de las sociedades en las Américas precoloniales como en las de la Europa pre-industrial confirma que la división del trabajo por género por lo general no creó ni reflejó desigualdades en la casa. Aunque existían jerarquías entre los géneros no se separaban ni jerarquizaban las labores de producción y reproducción. La economía fue economía familiar o de "toda la casa", lo que también implica que los espacios de trabajo de la mujer -la casa y sus alrededores-, así como los espacios de trabajo del hombre, formaban un conjunto. Los segundos no tenían más valor que los primeros. La separación jerárquica entre lo público y lo doméstico aún no existía.

Fue hasta la propagación y generalización de las relaciones de mercado que la división del trabajo por género fue transformada en una jerarquía clara y estable. Con la creciente mercantilización de la producción, los trabajos que generaban ingresos fueron más apreciados que las tareas caseras. Eso implicaba que se considerara más valioso el trabajo masculino que el femenino. Por el otro, esta jerarquización socio- 
funcional también condujo a jerarquizar los espacios de trabajo: al ser relacionados con ingresos monetarios, los lugares fuera del hogar se valuaron más alto, mientras que el hogar, como espacio de producción no mercantilizada y de la reproducción, fue expulsado del reino de la economía y convertido en un espacio "privado", no productivo. La casa dejó de ser un "lugar de trabajo", término nuevo que capta bien el imaginario geográfico del mundo laboral capitalista.

En suma, con la generalización de las relaciones de mercado, la valorización diferente de las actividades económicas, de las personas que las realizaron y de los lugares en los que las llevan a cabo, se convirtieron en un factor sistemático, estable y característico de la división del trabajo por género. Sin embargo, el significado de esta vinculación entre valor, por un lado, y actividad / grupo social / lugar, por el otro, va mucho más allá de la división del trabajo por género. Según Wallerstein $(1983,25)$, la correlación sistemática entre división del trabajo y valorización diferenciada de los trabajos, de los que los realizan y de los lugares en los que los hacen, no sólo surgió con el capitalismo, sino también es una de sus características clave. Equipara a este proceso de diferenciación y jerarquización sistemática de actividades económicas dentro de una división del trabajo, con la formación de centros y periferias; los procesos de periferiación se basan en las interrelaciones que existen entre la organización social y espacial de la división del trabajo. Para comprender porqué existen centros y periferias -y cómo llegaron a ser lo que son- hay que comprender el mecanismo básico de la división del trabajo capitalista: los procesos de producción se reparten en segmentos, y cada segmento tiene asignado tanto un valor económico específico (expresado en la parte del valor agregado total que esta secuencia logra aglutinar) como un grupo social y un lugar particular para ser desarrollado. En síntesis, un centro económico es un lugar que se distingue por un predominio de los procesos laborales que logran concentrar una buena parte del valor agregado, por lo cual los grupos sociales que los realizan son altamente remunerados. Por otro lado, las periferias se caracterizan por una mayoría de procesos laborales poco remunerados y por una menor generación de valor agregado.

\section{División y jerarquización de trabajo (II): ciudad y campo}

sión del trabajo entre ciudad y campo es el fundamento de toda división del trabajo y de todo desarrollo económico. Muchos autores también señalan que este intercambio entre ciudad y campo sea desigual. Marx (2009a, 429), p. ej., habla de una "antítesis" mientras Braudel reitera constantemente que la división del trabajo entre ciudad y campo no sólo es una necesidad irrefutable para el desarrollo de las ciudades ( $y$, por ende, para el capitalismo), sino también que estas relaciones siempre han sido desiguales en favor de la ciudad.

En su teorización sobre el papel que desempeña la ciudad en el surgimiento del capitalismo, Braudel comprende que el dinamismo de la ciudad se basa y no existe sin divisiones del trabajo jerárquicas: 
Towns are like electric transformers. They increase tension, accelerate the rhythm of exchange and constantly recharge human life. [...] [But] (t)he town only exists as a town in relation to a form of life lower than its own. There are no exceptions to this rule. [...] There is no town, no townlet without its villages, its scrap of rural life attached; no town that does not impose upon its hinterland the amenities of its market, the use of its shops, its weights and measures, its moneylenders, its lawyers, even its distractions. It has to dominate an empire, however tiny, in order to exist. (Braudel, 1985, 479-482, énfasis añadido)

En este punto resulta pertinente puntualizar dos aspectos: primero, que el término hinterland que Braudel emplea aquí, es tomado del alemán, por ser muy sugerente, ya que literalmente el término hinterland significa "el campo detrás", que evoca la idea de la marginalización del campo en el sentido de su vinculación indisoluble con la ciudad. Segundo, Braudel relaciona la capacidad de la ciudad de crear relaciones desiguales con el campo -y de vivir de ellas- con ciertas actividades económicas urbanas, a saber las que hoy se conocen como "servicios al productor" (como el prestamista o el abogado mencionados). Según Braudel las innovaciones financieras y su monopolización por parte de las élites urbanas son, sobre todo, herramientas clave que se utilizan para movilizar los recursos en el hinterland urbano y para su apropiación. Braudel detalla que el intercambio en las múltiples divisiones del trabajo es necesariamente desigual. Tomando la forma de "a chain of subordinations, each conditioning the others" (Braudel 1984, 48), puede asegurarse que la desigualdad de las relaciones socio-espaciales de las múltiples divisiones del trabajo es un factor constitutivo del capitalismo: "(T)here are inequalities, differences of voltage which make possible the functioning of the whole" (Braudel, 1984, 26).

Apreciaciones similares a las de Braudel sobre la desigualdad en las relaciones socio-espaciales en las relaciones entre ciudad y campo las encontramos también en la conceptualización de González Casanova (1965, 33), quien considera la división del trabajo como colonialismo interno, al asegurar que: "(T)he colonial structure resembles relations of domination and exploitation typical of the rural-urban structure of traditional society and of underdeveloped countries, insofar as a population integrated by several social classes (urban or colonial) dominates and exploits a population integrated by different classes (rural or colonized)".

\section{División y jerarquización de trabajo (III):} la economía mundial

En el pensamiento económico, la cuestión de la división del trabajo trans-

fronteriza preocupa a muchos autores. Mientras que en el mercantilismo la posición general fue negativa, para Smith la división del trabajo dentro de la fábrica se vincula con la escala del comercio exterior, ya que la división del trabajo en la fábrica sólo se desarrolla si el mercado (o sea, la demanda) es suficientemente grande. Una oportunidad limitada para el intercambio desalienta el refinamiento de esta división, y por lo tanto, el desarrollo económico. Según Smith, la solución para el problema de la estrechez del mercado se encuentra en el comercio exterior. 
En lo que se conoce como la teoría "Vent-for-surplus", Smith establece que al expandirse geográficamente (mediante el comercio exterior) se aumenta la división del trabajo en una escala más extensiva, lo cual permite continuar el aprovechamiento de sus ventajas. Sin embargo, para Smith no existe una distinción cualitativa entre comercio doméstico y comercio exterior: el último simplemente es la expansión del primero. La geografía es territorio, extensión y zona de captación, pero no diferenciación socio-espacial. En otras palabras, según Smith la división del trabajo transfronteriza no explota ni crea heterogeneidades geográficas en las áreas en las que se extiende: la geografía es entendida como cantidad, pero no como cualidad.

Por otra parte, en la obra de Marx sí encontramos evidencia de que él era consciente de que la división del trabajo transfronteriza sirve al capital para aprovechar (y, de hecho, para crear y después aprovechar) las diferenciaciones geográficas. Mientras Marx señaló con tino que la reestructuración socio-espacial de la producción ha sido en términos generales un prerrequisito para poner en marcha los procesos de acumulación de capital, cabe señalar que en lo que se refiere al tema de la división del trabajo, Marx abordó más el asunto de la transformación en el curso de la transición de la producción artesanal a la industria en los centros del capitalismo. Sin embargo, es bien sabido, y por lo mismo no se requiere profundizar aquí en este tema, que para Marx una expansión constante de la esfera de circulación era condición necesaria para el despegue de la industria, y en términos más generales, para la acumulación tanto como su resultado: "La tendencia a crear el mercado mundial está dada directamente en la idea misma del capital. Todo límite se le presenta como una barrera a salvar" (Marx, 2007, 360).

Lo que aquí interesa en particular es la importancia que Marx atribuye, aunque con cierta ambigüedad, a las diferenciaciones socio-espaciales en la división del trabajo transfronteriza (o como él la llamaba, en el comercio exterior o en el mercado mundial). En El capital, en la sección de sólo tres páginas dedicada al comercio exterior, apunta en relación con el capital invertido en las colonias que éste puede dar tasas de ganancia más altas "porque en esos lugares, en general, a causa de su bajo desarrollo, la tasa de ganancia es más elevada, y lo mismo, con el empleo de esclavos y culíes, etc., la explotación del trabajo" (Marx, 2009b, 304).

En la Miseria de la filosofía Marx también aborda el tema de la esclavitud "directa". que según él es una condición general que a la larga permite el surgimiento de la industria moderna. Sin embargo, Marx también reconoce su geografía específica. Relaciona ciertas actividades económicas, como el cultivo del algodón, con ciertos tipos de relaciones de producción y, más en concreto, del control del trabajo (esclavitud), y ambas con ciertas regiones (el Nuevo Mundo). También señala que esta diferenciación socio-espacial forma parte de la división del trabajo transfronteriza capitalista, en virtud de que ciertas regiones se integran en la división del trabajo precisamente por el predomino de algunas relaciones de producción. Sin esclavitud América no tiene justificación en el mapa del mundo capitalista: "La esclavitud ha dado su valor a las colonias [...] Suprimid la esclavitud y habréis borrado a Norteamérica del mapa de los pueblos" (Marx 1987, 69-70; énfasis añadido). 
En El capital, Marx también alude a las diferenciaciones socio-espaciales propias de la división del trabajo y su beneficio para los capitalistas. Apunta que la producción, fuertemente abaratada por la gran industria, es un arma poderosa para conquistar mercados externos, con una consecuencia en la estructura de la división del trabajo muy provechosa para la metrópoli:

(L)a baratura de los productos hechos a máquina y los sistemas revolucionados de trasporte y comunicación son armas para la conquista de mercados extranjeros. Al arruinar el producto artesanal de éstos, la industria maquinizada los convierte forzadamente en campos de producción de su materia prima. Así, por ejemplo, las Indias Orientales han sido constreñidas a producir algodón, lana, cáñamo, yute, añil, etc., para Gran Bretaña. [...] transformándolos [a los países extranjeros; C. P.] en semilleros de materias primas para la metrópoli, como se transformó por ejemplo a Australia en un centro de producción lanera. Se crea así una nueva división internacional del trabajo, adecuada a las principales sedes de la industria maquinizada, una división que convierte a una parte del globo terrestre en campo de producción agrícola por excelencia para la otra parte, convertida en campo de producción industrial por excelencia (Marx 2009a, 549-550; énfasis añadido).

Aunque utilice otras palabras, Marx destaca un proceso que hoy día se conoce como "la producción del espacio" (Soja, 1989; Harvey, 2001). Se trata de un proceso socioespacial que transforma las relaciones de producción y crea nuevas geografías productivas. Las colonias son preparadas y modificadas para servir a los intereses de la metrópoli. Parte del proceso de la producción de espacios conlleva, como afirma Marx, que las relaciones de producción predominantes en una sociedad y región lleguen a impregnarlas. En una carta a Annenkov Marx pregunta (1987, 135; énfasis añadido): "Toda la organización interior de los pueblos, todas sus relaciones internacionales, ¿no son acaso la expresión de cierta división del trabajo?, ¿no deben cambiar con los cambios de la división del trabajo?"2

El concepto de que la organización "interna" de una sociedad dependa críticamente de su manera de integración "externa" en la división del trabajo capitalista mundial fue convertido en el foco de atención por Wallerstein, siendo esta idea una de las esencias de su Análisis de sistema mundo, en donde argumenta que el acontecimiento decisivo para que surja el capitalismo a partir del siglo XV fue la organización de una división del trabajo transfronteriza con varios modos de organización de las relaciones de producción.

2 La ambigüedad mencionada surge del hecho de que Marx sitúa esta diferenciación socioespacial en algunas regiones en una división del trabajo marcada por relaciones laborales totalmente diferentes que en otras regiones, sólo que en tiempos de acumulación originaria. Como apunta en El capital: "El descubrimiento de las comarcas auríferas y argentiféras en América, el exterminio, esclavización y soterramiento en las minas de la población aborigen, la conquista y saqueo de las Indias Orientales, la transformación de África en un coto reservado para la caza comercial de pieles-negras, caracterizan los albores de la era de producción capitalista. Estos procesos idílicos constituyen factores fundamentales de la acumulacion originaria" (Marx, 2009a, 939). 
Esta división del trabajo global surgió a partir de 1450; tuvo su centro en el noroeste de Europa, e incluyó como su periferias Europa del Este, y las regiones de Mesoamérica y América del Sur. Y lo fundamental en nuestro análisis es no perder de vista que los productores en las distintas regiones no sólo aportaron productos diferentes, sino que también los produjeron en condiciones muy distintas. En Europa occidental, y sobre todo en su parte noroeste, los productores y trabajadores se especializaron cada vez más en industrias y servicios (sobresalen los financieros y los de comercio), mientras que Europa del Este fue incluida en la división del trabajo capitalista como granero y como proveedor de madera. A pesar de que esta producción de granos y madera haya sido de mercado, se llevó a cabo no por la vía del trabajo asalariado, como se ha convertido en la norma en las economías del noroeste de Europa, sino mediante la re-propagación de relaciones laborales semi libres, o no-libres, la llamada "segunda servidumbre". Por otro lado, las regiones de Mesoamérica y América del Sur fueron integradas en la división del trabajo capitalista como proveedores de plata, oro y azúcar, en cuya producción se utilizaba, en la mayoría de los casos, diferentes formas de trabajo forzado. Sin embargo, aunque se hubiera tratado de trabajadores no-libres, producían como sus iguales en Europa del Este, para el mercado de la economía mundial emergente. Mientras Europa del Este alimentaba a Europa Occidental (y liberó de esta forma a los campesinos para que pudieran realizar trabajos industriales), la función principal de las colonias españolas y portuguesas fue proporcionar liquidez a los mercados en expansión (Wallerstein 1974).

Esta diferenciación socio-geográfica entre lo que Wallerstein llama centro y periferia tiene como sustento no sólo la especialización económica específica, sino los diferentes modos de control del trabajo y el desarrollo diferenciado de estructuras de clase y en fuerza del Estado. Las regiones -y sociedades- que lograron convertirse en centros de la economía mundial se especializaron en actividades económicas con mayor valor agregado y mejor remuneración, y por lo mismo desarrollaron burguesías fuertes, además de relaciones laborales caracterizadas por el trabajo asalariado, todo lo cual se tradujo en estados fuertes. Por el contrario, las regiones y sociedades que terminaron siendo periferias se caracterizaron por sus actividades económicas de poco valor agregado, trabajos mal remunerados; por élites más feudales que capitalistas; por relaciones laborales dominadas por varias formas de trabajo forzado, y por estados débiles o incluso ausentes (Wallerstein 1983). Lo interesante aquí es que según Wallerstein estas dimensiones funcionales, sociales y geográficas de la división del trabajo capitalista se encuentran necesariamente interrelacionadas.

El punto clave para comprender la razón por la que según Wallerstein la geografía de la división del trabajo importa para su jerarquización es que la imposición de las distinciones en actividades económicas, remuneración, condiciones laborales, etcétera, resulta políticamente más fácil si se logran separar espacialmente los clusteres socio-económicos. No existe, por ejemplo, ningún factor ecológico que hubiera podido impedir cultivar trigo a gran escala en el noroeste de Europa para alimentar las ciudades y sus actividades industriales y de comercio florecientes. Sin embargo, imponer, como se hizo en Polonia, servidumbre a los campesinos en el noroeste de Europa hubiera sido políticamente contenido y por lo mismo difícil, si no es que 
imposible de lograr, ya que el trabajo asalariado en las ciudades en esta zona se estaba extendiendo con rapidez. Por otra parte, comprarles el trigo a campesinos libres en el noroeste de Europa hubiera encarecido enormemente la reproducción de los trabajadores urbanos y de esta manera se hubiera frenado la acumulación. Así las cosas, políticamente fue más conveniente separar en lo geográfico la servidumbre del trabajo asalariado y de esta manera relacionar ciertas regiones con determinadas características económicas y sociales.

La correlación entre zonas y circunstancias socio-económicos es,

Las relaciones socioespaciales de una región dependen de manera directa de su inserción en las relaciones socioespaciales de la división del trabajo mundial capitalista. Es así como nacen las regiones "desarrolladas" 0 "subdesarrolladas" entonces, en gran parte producto social. La aparición de dichas "geographico-economic zones" (Wallerstein 1983, 28) corresponde a una dinámica de segmentación socio-espacial de la división del trabajo capitalista uniforme. Esta segmentación la muestra como la formación de centros y periferias. Como bien apunta Harvey (2006, xVIII): "[...] all of this would also entail the production of spatial configurations and 'regions' of activity (through, for example, capital assets being embedded in the built environments of cities, through territorial divisions of labour, etc.) and that regional class alliances and forms of territorial organization and governance would emerge around such spatial structures".

En otras palabras, las relaciones socio-espaciales de una región dependen de manera directa de su inserción en las relaciones socio-espaciales de la división del trabajo mundial capitalista. Es así como nacen las regiones "desarrolladas" o "subdesarrolladas", y por lo cual el análisis de las geografías de las relaciones de producción resulta imprescindible para comprender las dinámicas del capitalismo. Para Massey (1995, 56): “The 'order of analysis', then, [...] should not be first the aspatial, or the 'aggregate' [...] and then the distribution over space; first production and only then location. 'Geography' should be part of the specification from the beginning. The reproduction of social and economic relations and of the social structure takes place over space".

\section{Reconciliar la ciencia económica y la geografía sí es posible}

Paul Krugman (2013) coquetea con la geografía económica, al adular que

"(it is) a field full of empirical insights, good stories, and obvious practical importance, lying neglected right under our noses because nobody had seen a good way to formalize it". Ahora bien, su intento de formalizar y modelar todos estos conocimientos e historias para reconciliar la ciencia económica con la geografía fracasó. Es precisamente porque Krugman insiste en que modelar sea el único método suficientemente científico para un análisis espacial de la economía, un acercamiento entre las disciplinas de la economía y de la geografía resulta muy difícil. El geógrafo Martin asegura con resignación que "un diálogo de sordos parece más probable" $(2013,255)$.

Sin embargo, aún hay esperanza. La presente discusión sobre la división del trabajo y su inherente espacialidad ha mostrado ser una vía para re-insertar el enfoque geográfico al análisis económico. La división del trabajo, entendida no como mero principio económico sino como resultado de prácticas y relaciones concretas entre 
múltiples actores, no sólo implica que ciertas labores sean realizadas por determinadas personas o grupos, sino también que se lleven a cabo en espacios específicos acordes a estas tareas y personas o grupos. En otros términos, la división del trabajo es una forma de organizar la producción en el espacio y utilizándolo para crear de este modo geografías económicas específicas y diferenciadas. Para hacer visibles las interrelaciones entre las dimensiones funcionales, sociales y geográficas de la división del trabajo he utilizado el concepto "relaciones socio-espaciales", el cual establece que la forma geográfica de la división del trabajo equivale a "la organización espacial de las relaciones de producción" (Massey, 1995, 7).

Para unir a economistas y geógrafos en un análisis sobre la organización espacial de las relaciones de producción, un tema ideal es la globalización, ya que los procesos relacionados con ella se vinculan con las relaciones socio-espaciales, mismas que atan, estructuran y dan jerarquía a las divisiones del trabajo en diferentes escalas. Unamos nuestros esfuerzos a fin de comprenderlo.

\section{Bibliografía}

Asuad Sanen, N. 2014, Pensamiento económico y espacio, México, Universidad Nacional Autónoma de México (Colección Economía Regional y Urbana, 1).

BLAUG, M. 1985, Economic Theory in Retrospect, Cambridge, Cambridge University Press. 1984, The Perspective of the World (Civilization and Capitalism: 15th-18th Century, vol.

3. Londres, Collins.

1985. The Structures of Everyday Life. The Limits of the Possible, vol. 1, Civilization and Capitalism: 15th-18th Century, Londres, Collins.

CIPOLLA, C. 1993, Before the Industrial Revolution: European Society and Economy, 1000-1700, Londres, Routledge.

HARVEY, D. 2006, The Limits to Capital, Londres, Verso.

2001, Spaces of Capital. Towards a Critical Geography, Nueva York, Routledge.

Hopkins, T. e I. WAllerstein, 1994, "Commodity Chains in the World Economy Prior to 1800", en Review, 10 (1): 157-170.

ISARD, W. 1956, Location and Space Economy: A General Theory Relating to Industrial Location, Market Areas, Land Use, Trade, and Urban Structure, Cambridge, MIT Press.

KRUGMAN, 1998, "What's New About the New Economic Geography?", en Oxford Review of Economic Policy, 14: 7-16.

2000. "Where in the World Is the 'New Economic Geography?", en G. CLARK, M. FELDMAN

y M. S. GeRTLER (eds.), The Oxford Handbook of Economic Geography, Oxford, Oxford University Press: 49-60.

2013. "Crib Sheet: How I Work (Self-indulgent)", en New York Times, 5 de julio de 2013. En: [http://krugman.blogs.nytimes.com/2013/07/05/crib-sheet-how-i-work-selfindulgent/?_r=0]. Consultado el 27 de agosto de 2015.

y R. WELLS, 2012, Macroeconomics, Worth Publishers. 
MARTIN, R., 2013, “El nuevo ‘giro geográfico’ en Economía. Algunas reflexiones críticas”, en M. VALDIVIA LÓPEZ, J. DELGADILlO MAcíAs (eds.), La geografía y la economía en sus vínculos actuales: una antología comentada del debate contemporáneo, Cuernavaca, México, Universidad Nacional Autónoma de México: 235-264.

MARX, K., 1987, Miseria de la filosofía. Respuesta a la "Filosofía de la miseria" de Proudhon [1846-1847], México, Siglo XXI Editores.

2007, Elementos fundamentales para la critica de la economía política (Grundrisse) [18571858], vol. 1. México, Siglo XxI Editores.

2009a , El capital. Crítica de la economía política, Libro l [1887], México, Siglo XXI Editores. 2009b, El capital. Crítica de la economía política, Libro III [1887], México, Siglo XXI Editores.

MASSEY, D. 1995, Spatial Divisions of Labor, Londres, Macmillan.

Matthael, J. A. 1999. "Patriarchy", en J. Peterson y M. LeWIS (eds.), The Elgar companion to feminist economics. Cheltenham, Edward Elgar: 592-599.

Medema, S. G. y W. J. Samuels (eds.). 2003. The History of Economic Thought: A Reader. Londres, Routledge.

PORTES, A. 2000. "Immigration and the Metropolis: Reflections on Urban History", en Journal of International Migration and Integration, 1 (2): 153-175.

SмITH, A. 1776. An Inquiry into the Nature and Causes of the Wealth of Nations. Londres, Methuen \& Co.

SOJA, E. 1989. Postmodern Geographies: The Reassertion of Space in Critical Social Theory. Londres, Verso.

SuN, G. 2005. Readings in the Economics Division of Labor: The Classical Tradition. Nueva Jersey, World Scientific Publishing.

SuN, G. 2012. The Division of Labor in Economics: A History. Nueva York, Routledge.

Valdivia López, M. y J. Delgadillo Macías. 2013. "Presentación”, en M. Valdivia López y J. Delgadillo MAcíAs (eds.), La geografía y la economía en sus vínculos actuales: una antología comentada del debate contemporáneo. Cuernavaca, Universidad Nacional Autónoma de México: 11-24.

WALLERSTEIN, I. 1974a. "The Rise and Future Demise of the World Capitalist System. Concepts for Comparative Analysis", en Comparative Studies in Society and History, 16 (4): 387-415.

1974. The Modern World System: Capitalist Agriculture and the Origins of the European World-Economy in the Sixteenth Century. Nueva York, Academic Press. 1983. Historical Capitalism. Londres, Verso. 